\title{
Estrutura de Classes, Educação e Queda da Desigualdade de Renda (2002-2011)*
}

\author{
Pedro Herculano Guimarães Ferreira de Souza ${ }^{1}$ \\ Flavio Alex de Oliveira Carvalhaes ${ }^{2}$ \\ ${ }^{1}$ Instituto de Pesquisa Econômica Aplicada (Ipea), Brasília, Brasil. \\ ${ }^{2}$ Centro de Pesquisa e Documentação Histórica Contemporânea do Brasil da Fundação \\ Getulio Vargas (CPDOC-FGV), Rio de Janeiro, Brasil.
}

\section{INTRODUÇÃO}

\begin{abstract}
queda da desigualdade de renda per capita é um dos fenômenos A mais estudados da última década no Brasil, com alguns consensos bem estabelecidos. Um dos mais importantes diz respeito às causas desse fenômeno: se, por um lado, a expansão das transferências públicas, como as do Programa Bolsa Família e do Benefício de Prestação Continuada, desempenhou um papel relevante, por outro, não há dúvidas de que o mercado de trabalho foi o principal fator por trás da queda da desigualdade (Soares, 2006; Barros, Franco e Mendonça, 2007a; Hoffmann, 2007).
\end{abstract}

Há argumentos de diversos tipos para explicar por que o mercado de trabalho tornou-se menos desigual, desde investigações sobre a relativa homogeneização da composição etária da população e, portanto, diminuição dos retornos para a experiência (Barros et al., 2007) até análises de mudanças na segmentação setorial e espacial no Brasil (Ferreira et al., 2006; Barros, Franco e Mendonça, 2007b). No entanto, maior peso costuma ser dado às mudanças na composição educacional e nos retornos à educação da força de trabalho como grandes causas dessa queda recente (Menezes-Filho, Fernandes e Picchetti, 2006; Barros, Franco e Mendonça, 2007a).

\footnotetext{
* Os autores agradecem a Marcelo Medeiros, José Alcides Figueiredo Santos, Sergei Soares e Luis Felipe Batista Oliveira pelos comentários.
}

DADOS - Revista de Ciências Sociais, Rio de Janeiro, vol. 57, no-1, 2014, pp. 101 a 128. 
O propósito deste texto é saber em que medida um ponto de vista tipicamente sociológico - da análise de classes - ajuda a iluminar o fenômeno e como ele se relaciona com os fatos já estabelecidos pela literatura da área. Mais especificamente, o artigo tem como objetivo investigar como mudou a estrutura de classes nesses últimos anos, o quanto da queda da desigualdade ela é capaz de explicar - tanto de uma perspectiva estática quanto dinâmica - e como tais mudanças se relacionam com os efeitos identificados da educação na desigualdade. Afinal, uma maior oferta de trabalhadores mais educados pode ensejar uma série de fenômenos distintos que colaborariam para a queda da desigualdade, como mudanças nos tamanhos relativos das classes, compressão salarial dentro de cada classe ou mudanças nos salários médios relativos entre classes.

Para isso, o texto está organizado da seguinte maneira: além da presente Introdução, a segunda seção trata de aspectos gerais da ligação entre classe, educação e a estrutura da desigualdade; a terceira define a tipologia de classes utilizadas e esclarece a metodologia empregada nas decomposições; a quarta seção documenta as principais mudanças no mercado de trabalho brasileiro entre 2002 e 2011; a quinta apresenta decomposições univariadas estáticas e dinâmicas da desigualdade tal como medida pelo GE(0); a sexta seção traz as decomposições multivariadas via regressão a partir da abordagem de Fields (2003); a sétima e última seção reúne nossas principais conclusões.

\section{A ESTRUTURA DA DESIGUALDADE: CLASSE E EDUCAÇÃO}

Grosso modo, duas perspectivas teóricas são tradicionalmente acionadas na investigação da desigualdade de renda no mercado de trabalho. A primeira dá destaque às características da oferta de trabalho, com a motivação básica de compreender a relação entre educação, experiência e renda. A segunda tem como foco principal a relação entre a estrutura de classes e a desigualdade. Tipicamente, essas perspectivas coincidem com divisões disciplinares, sendo a primeira mais comum entre economistas e a segunda entre sociólogos, apesar de haver uma relativa sobreposição entre os debates.

Normalmente, do ponto de vista da sociologia, a principal objeção ao primeiro tipo de explicação está no foco excessivo dado pelas análises empíricas às características individuais da oferta da mão de obra, em detrimento de aspectos estruturais (Sorensen, 1996). Assim, a forma privilegiada de alcançar essa dimensão seria através da atenção à di- 
mensão ocupacional ou da análise de classes. Instituições do mercado de trabalho e da demanda pelo trabalho seriam fundamentais para compreender os diferenciais de rendimento.

Nesta perspectiva, algumas ocupações ou classes, menos especializadas e mais abertas à substituição de trabalhadores, seriam menos protegidas e mais sujeitas às flutuações ligadas à composição da oferta de trabalho. Outras, devido às suas próprias idiossincrasias (complexidade das tarefas, interdependência na cadeia produtiva, entre outras), têm outro tipo de dinâmica, mais fechada (Parkin, 1979; Sorensen e Kalleberg, 1981; Goldthorpe, 2000). É a atenção a esse tipo de características um dos pontos centrais dos trabalhos ligados à análise de classes, em seus esforços para agrupar as ocupações em tipologias, independente dos princípios analíticos adotados - tipo de contrato de trabalho, no caso neoweberiano, e relação com meios de produção e exploração, no caso neomarxista (Breen e Rottman, 1995; Wright, 2000; Goldthorpe, 2000).

Mas como a estrutura de classes se ligaria a mudanças na tendência da desigualdade de renda? Há três tipos de processos possíveis. Primeiro, pode haver mudanças estruturais no mercado de trabalho que levariam a uma nova composição de classes em termos de tamanhos relativos, ou seja, um efeito de alocação da força de trabalho. Segundo, pode haver mudanças nas médias relativas dos salários entre classes, seja por restrições políticas, como em parte ocorreu no Brasil dos anos 1960 e 1970 (Hoffmann e Duarte, 1972; Hoffmann, 1973), seja por outros fatores, como flutuações na demanda por trabalho, ações de licenciamento, certificação ou outras (Grusky e Sorensen, 1998; Weeden, 2002). Terceiro, pode haver mudanças na desigualdade intraclasses, causadas, por exemplo, por mudanças na remuneração de outras características, que dizem respeito, por exemplo, aos arranjos institucionais que regulam o mercado de trabalho; a diferenças entre setores heterogêneos, entre outras. Neste artigo, tentaremos ponderar entre esses três diferentes mecanismos e sua importância na tendência da desigualdade de renda no Brasil entre 2002 e 2011.

\section{CLASSIFICAÇÕES E MÉTODOS DE DECOMPOSIÇÃO}

\section{a) Tipologia de Classes}

Neste estudo, a estrutura de classes brasileira será entendida a partir da tipologia formulada por Santos (2005), que é inspirada no esquema 
neomarxista de Erik Olin Wright. Em Wright, a distribuição desigual de poderes e direitos sobre os recursos produtivos dá origem à estrutura de classes, que, por sua vez, condiciona as oportunidades de vida disponíveis para os indivíduos. Para ele, cada período histórico é marcado por uma forma principal de exploração, ligada à distribuição desigual de um ativo produtivo central. No capitalismo, isso corresponderia à distribuição desigual da propriedade dos meios de produção, mas a exploração e as relações de classes contemporâneas também seriam estruturadas em torno da posse/não posse de outros ativos produtivos, como as habilidades/credenciais e os ativos organizacionais (Wright, 2000; 2005).

A classificação de Santos (2005) procura refinar a de Wright e adaptá-la para a realidade brasileira, com seu grande e heterogêneo contingente de trabalhadores por conta própria e alcance restrito do assalariamento formal. A principal fonte de informações para a tipologia é a posição na ocupação, que discrimina os trabalhadores em relação à posse de capital: empregadores, empregados e trabalhadores por conta própria. Em seguida, cada uma das três categorias é desagregada de acordo com os tipos e o volume dos ativos possuídos, o que envolve o recurso a variáveis auxiliares como o setor da atividade, o título ocupacional, entre outras.

Dessa forma, a força de trabalho é classificada em um total de 13 classes: os empregadores dividem-se em capitalistas e fazendeiros (a classe mais privilegiada e com maior controle sobre o capital) e pequenos empregadores; os trabalhadores por conta própria dividem-se em agrícolas, não agrícolas, precários (sem acesso a capital) e especialistas autoempregados (segmento privilegiado pelo acesso a habilidades e que também inclui pequenos empregadores cujo título ocupacional denote conhecimento perito); finalmente, os empregados repartem-se em gerentes (dotados de qualificação e autoridade), empregados especialistas (dotados de qualificação), empregados qualificados (em geral técnicos de nível médio e outros trabalhadores com níveis mais restritos de qualificação), supervisores (dotados de autoridade), trabalhadores típicos (grosso modo, é o trabalho proletarizado "padrão"), trabalhadores elementares (com níveis mínimos de especialização e responsáveis pelas tarefas mais simples), e trabalhadores domésticos (Santos, 2005). Como os militares não são classificados na tipologia de Santos, eles foram excluídos de todas as análises. 
O esquema de classificação socioeconômica de Santos é atraente porque é bastante canônico: ao seguir de perto a lógica de Wright, acaba sendo parecido também com as tipologias de Goldthorpe e seus associados, bastante utilizadas em estudos empíricos sobre desigualdade e mobilidade social (Erikson e Goldthorpe, 1992; Goldthorpe, 2000). Afinal, as bases teóricas dos esquemas de Wright e Goldthorpe são distintas, mas esses esquemas convergem na prática quando operacionalizados por meio de títulos ocupacionais. Além disso, a vantagem da classificação de Santos está em ser elaborada especificamente para o Brasil, o que se reflete tanto no plano conceitual quanto na sua operacionalização: no primeiro caso, são feitas distinções importantes em categorias heterogêneas de trabalhadores precários que não seriam captadas em tipologias internacionais; no segundo caso, não é necessário fazer nenhuma tradução ou adaptação ad hoc para compatibilizar classificações internacionais com as variáveis específicas das pesquisas brasileiras.

Mais ainda, a classificação de Santos é interessante porque, embora se preocupe essencialmente com a estrutura da demanda por trabalho, toma como um dos ativos estruturantes das posições de classes justamente a "qualificação", o que abre a possibilidade de diálogo com abordagens voltadas para a oferta de trabalho. Naturalmente, existem diferenças teóricas fundamentais entre os dois tipos de abordagens, mas, se tomarmos a educação como proxy razoável da qualificação, podemos então testar empiricamente a hipótese de que mesmo estimativas conservadoras da influência da estrutura de classes sobre a desigualdade (isto é, estimativas que supõem que a teoria do capital humano está "correta" e avaliam a influência das classes líquida do efeito da educação) reiteram a importância de ambos efeitos sobre o nível e a trajetória da desigualdade e, em última instância, enriquecem nosso entendimento sobre as mudanças ocorridas no Brasil na última década.

\section{b) Fontes de Dados}

Todos os dados utilizados neste texto são provenientes da Pesquisa Nacional por Amostra de Domicílios (PNAD), realizada anualmente exceto em anos censitários - pelo Instituto Brasileiro de Geografia e Estatística (IBGE). Como a PNAD 2002 não cobria as áreas rurais dos estados da Região Norte (exceto Tocantins), estas áreas também foram excluídas da PNAD 2011. 
Todas as análises baseiam-se apenas no trabalho principal dos indivíduos, excluindo trabalhadores não remunerados e militares, bem como indivíduos cujo status no domicílio era de empregado doméstico, pensionista e afins. Nossas análises de sensibilidade indicam que o uso da renda horária ou a limitação da análise apenas aos que trabalham 20 horas ou mais não alteram substantivamente os resultados do trabalho. A Tabela 1 apresenta informações básicas sobre as amostras para 2002 e 2011:

\section{Tabela 1}

Informações Básicas sobre as Amostras das PNADs

(Brasil, 2002 e 2011)

\begin{tabular}{l|r|r}
\hline & 2002 & \multicolumn{1}{|c|}{2011} \\
\hline Amostra Total & 385.431 & 358.919 \\
\hline Pessoas & 173,254 & 195,243 \\
População expandida (milhões) & & \\
\hline População civil ocupada remunerada & 149.983 & 146.922 \\
Pessoas & 67,759 & 81,009 \\
População expandida (milhões)
\end{tabular}

Fonte: Microdados da PNAD (2002 e 2011), exclusive áreas rurais de Acre (AC), Amazonas (AM), Amapá (AP), Pará (PA), Rondônia (RO) e Roraima (RR).

Para facilitar a interpretação, todos os rendimentos foram deflacionados para junho de 2013. O deflator aplicado à renda foi o Índice Nacional de Preços ao Consumidor (INPC), ajustado de acordo com a sugestão de Corseuil e Foguel (2002).

\section{c) Medidas e Decomposições da Desigualdade}

A principal medida de desigualdade empregada neste trabalho é o GE(0), isto é, o índice de entropia generalizada com $\alpha=0$, também conhecido como L de Theil. O GE(0), assim como os demais indicadores da classe de medidas de entropia, possui as propriedades da Curva de Lorenz e é aditivamente decomponível: a desigualdade total é a soma da desigualdade dentro dos grupos e da desigualdade entre os grupos (Shorrocks, 1980).

O GE(0) é mais atraente do que os demais indicadores de entropia justamente porque permite uma interpretação contrafactual: o componente entre grupos é exatamente igual a quanto a desigualdade total cairia caso as rendas médias de todos os grupos fossem igualadas. Por 
isso, utilizamos o GE(0) nas decomposições da desigualdade. Além disso, o índice também se presta a decomposições dinâmicas de forma mais simples do que outras medidas de entropia. Em termos de sua função de bem-estar, o GE(0) dá mais peso à cauda inferior da distribuição, isto é, aos mais pobres.

A decomposição estática do GE(0) por $k$ subgrupos é dada por:

$G E(0)=\sum_{j=1}^{k} f_{j} G E(0)_{j}+\sum_{j=1}^{k} f_{j} \log \left(\frac{1}{\lambda_{j}}\right)$

Onde o primeiro termo representa o componente intragrupos e o segundo, o entre grupos; $f_{j}$ é a participação relativa do subgrupo $j$ na população; $G E(0)$, é o índice de desigualdade para o subgrupo $j$; e $\lambda_{j}$ é a renda média relativa $\left({ }^{\mu}{ }^{i} / \mu\right)$ do subgrupo $j$.

A decomposição dinâmica - isto é, comparando dois pontos no tempo - do GE(0) é obtida por:

$\Delta G E(0) \cong \sum_{j=1}^{k} \overline{f_{j}} \Delta G E(0)_{j}+\sum_{j=1}^{k} \Delta f_{j} \overline{G E(0)_{j}}+\sum_{j=1}^{k} \Delta f_{j}\left[\overline{\lambda_{j}}-\overline{\log \left(\lambda_{j}\right)}\right]+\sum_{j=1}^{k}\left(\overline{v_{j}}-\overline{f_{j}}\right) \Delta \log \left(\mu_{j}\right)$

Onde $v_{j}$ é a participação do subgrupo $j$ na renda total; $\mu_{j}$ é a renda média do subgrupo $j$ e a barra horizontal indica a média entre os dois anos (Mookherjee e Shorrocks, 1982). O primeiro termo representa o efeito "puro", isto é, o efeito de mudanças nas desigualdades internas dos grupos na variação da desigualdade total; o segundo e o terceiro termos representam o efeito alocação, isto é, o quanto mudanças nos tamanhos relativos dos grupos contribuíram para variações na desigualdade total; finalmente, o terceiro termo representa o efeito "renda", ou seja, o quanto mudanças nas rendas médias relativas dos grupos contribuíram para variações na desigualdade total.

Para tornar o trabalho mais exaustivo, três outras medidas de desigualdade são ocasionalmente mencionadas: recorremos também ao coeficiente de Gini e ao GE(1) - ou T de Theil - para medir a robustez da queda da desigualdade e ao índice de dissimilaridade para avaliar mudanças na distribuição da população ocupada por classes e níveis educacionais.

As duas primeiras medidas são bastante conhecidas e dispensam apresentações. Já o índice de dissimilaridade é uma medida não paramétri- 
ca do grau em que duas distribuições com a mesma classificação em categorias diferem entre si. Para $n$ categorias, sua fórmula é dada por:

$$
I D=\frac{1}{2} \sum_{i=1}^{n}\left|\frac{x_{i}}{X}-\frac{y_{1}}{Y}\right|
$$

Onde $x_{i}$ e $y_{i}$ correspondem ao número de casos na i-ésima categoria de cada distribuição e $X$ e $Y$ correspondem ao total de casos das duas distribuições. O índice representa o percentual de casos da segunda distribuição que devem ser reclassificados para que ela se torne idêntica à primeira e, portanto, varia entre $0 \%$ e $100 \%$.

As decomposições multivariadas da desigualdade são obtidas pelo método proposto por Fields (2003) com base em regressões e respondem a dois tipos de perguntas: dada uma regressão qualquer para determinar a renda individual, trata-se de saber, primeiro, quanto cada variável explica da desigualdade total e, segundo, quanto cada variável explica de mudanças na desigualdade entre dois pontos no tempo.

Matematicamente, considere uma função geradora de renda tradicional:

$y=X \beta+\varepsilon$

Onde $y$ é um vetor $n x 1$ do logaritmo dos rendimentos, $X$ é uma matriz $n x(k+1)$ de características dos indivíduos, incluindo um termo para a constante, $\beta$ é um vetor $(k+1) \times 1$ de coeficientes e $\varepsilon$ éo vetor $n \times 1$ de resíduos. O modelo estimado a partir da amostra pode ser reescrito como:

$$
\begin{aligned}
y & =b_{0}+b_{1} x_{1}+\ldots+b_{k} x_{k}+u \\
& =b_{0}+\hat{z}_{1}+\ldots+\hat{z}_{k}+\hat{z}_{k+1}
\end{aligned}
$$

A variância de y é decomponível como a soma das covariâncias entre as variáveis compostas e o próprio y:

$$
\sigma^{2}(y)=\sum_{j=1}^{k+1} \operatorname{cov}\left(\hat{z}_{j}, y\right)
$$

O percentual da variância do logaritmo da renda explicado por cada variável é dado por:

$s_{j}(y)=\frac{\operatorname{cov}\left(z_{j}, y\right)}{\sigma^{2}(y)}$ 
Com:

$\sum_{j=1}^{k+1} s_{j}(y)=100 \%$

Se o último elemento do somatório for excluído, o percentual explicado é equivalente ao $\mathrm{R}^{2}$ da regressão inicial:

$\sum_{j=1}^{k} s_{j}(y)=R^{2}$

Embora seja atraente, esta decomposição é limitada por se aplicar à variância do logaritmo da renda, uma medida de desigualdade insatisfatória porque não respeita o princípio das transferências (Foster e Ok, 1999). Fields lança mão do trabalho de Shorrocks (1982) para mostrar que os pesos relativos de cada variável obtidos pelas fórmulas acima independem da medida de desigualdade utilizada desde que sejam assumidos alguns pressupostos razoavelmente canônicos, como continuidade, simetria, decomponibilidade e independência do nível de desagregação, entre outros (Fields, 2003). A contribuição de um fator para a desigualdade deve ser igual a zero quando todos os recipientes receberem a renda média daquele fator.

Na prática, isso significa que o peso na desigualdade total derivado para cada fator $z_{k}$ é o mesmo das medidas de desigualdade mais populares, como o índice de Gini, os índices de entropia e o índice de Atkinson, entre outros. Vale lembrar, no entanto que, neste caso, a variável dependente da decomposição de Fields é o logaritmo da renda, e não a própria renda. Ou seja, os pesos obtidos são válidos, por exemplo, para a decomposição do logaritmo da renda, mas não são diretamente comparáveis com a decomposição da própria renda.

Tais decomposições estáticas da desigualdade podem também ser facilmente estendidas para a comparação entre dois pontos no tempo, embora nesse caso os pesos atribuídos a cada variável dependam da medida de desigualdade escolhida. Para uma dada medida de desigualdade $I$, as mudanças verificadas entre $t$ e $t+1$ podem ser decompostas em:

$I_{t+1}-I_{t}=\sum_{j=1}^{k+1}\left(S_{j, t+1} I_{t+1}-S_{j, t} I_{t}\right)$ 
Assim, a contribuição relativa de cada fator para a variação na desigualdade é dada por:

$\Pi_{j}\left(I_{t, t+1}\right)=\frac{S_{j, t+1} I_{t+1}-S_{j, t} I_{t}}{I_{t+1}-I_{t}}$

Com:

$\sum_{j=1}^{\kappa+1} \Pi_{j}\left(I_{t, t+1}\right)=100 \%$

As fórmulas acima permitem decomposições multivariadas da desigualdade. Em particular, permitem uma tentativa de isolar os efeitos de mudanças educacionais dos demais componentes associados à estrutura e classes. Há, no entanto, uma limitação nessas fórmulas: os "fatores" da decomposição são os produtos de $b_{k} x_{k}$ : ou seja, não temos como separar facilmente as variações nas quantidades (a distribuição dos $x_{k}$ ) das variações nos preços $\left(b_{k}\right)$, ao contrário do que ocorre com as decomposições do GE(0).

Em suma, os dois tipos de decomposições cumprem papéis distintos. No caso do GE(0), são decomposições univariadas - isto é, decomposições que só consideram uma variável e, por isso, medem sua contribuição bruta para a desigualdade - mas que permitem, com grande facilidade, investigar detalhes das mudanças ${ }^{1}$. No caso das regressões, a decomposição multivariada permite avaliar as contribuições "líquidas" de cada variável para a desigualdade total, ou seja, a contribuição dada por uma variável mesmo quando mantemos constante um conjunto importante de controles. Como sempre, não há exatamente uma decomposição "melhor" do que outra; cada uma tem seus pressupostos e seus limites e o uso de ambas serve para tornar mais robustas as evidências apresentadas.

Por fim, vale apenas lembrar que tanto as decomposições univariadas do GE(0) quanto as decomposições multivariadas via regressão são feitas a partir de medidas sintéticas da desigualdade, isto é, medidas que resumem toda a informação sobre as distribuições de renda em um único número (ver discussão em Fortin, Lemieux e Firpo, 2011). Com isso, explicar o que acontece em pontos específicos da distribuição por exemplo, entre os mais pobres ou entre os mais ricos - está fora do escopo deste artigo. 


\section{QUEDA DA DESIGUALDADE E MUDANÇAS NO MERCADO DE TRABALHO ENTRE 2002 E 2011}

Depois de grande estagnação, em especial nas regiões metropolitanas, durante boa parte dos anos 1990, o mercado de trabalho brasileiro apresentou comportamento muito positivo a partir de meados da década passada. Todos os principais indicadores melhoraram entre o biênio 2002-2003 e 2011. A Tabela 2 traz dados relativos à força de trabalho ocupada e remunerada, exclusive militares, que também não são contabilizados nas tabelas seguintes.

Tabela 2

Estatísticas Selecionadas sobre a Participação no Mercado de Trabalho da População Ocupada Remunerada

(Brasil, 2002 e 2011)

\begin{tabular}{l|c|c|c}
\hline & $\mathbf{2 0 0 2}$ & $\mathbf{2 0 1 1}$ & Variação \\
\hline Formalização (\%) & 53,4 & 65,0 & $11,6^{* * * *}$ \\
Renda do trabalho principal (R\$ 2013) & 1.193 & 1.454 & $21,9^{* * *}$ \\
Horas trabalhadas por semana & 42,3 & 40,7 & $-3,9^{* * * *}$ \\
Gini & 0,555 & 0,493 & $-112^{* * *}$ \\
GE(0) & 0,568 & 0,440 & $-22,5^{* * *}$ \\
GE(1) & 0,635 & 0,519 & $-18,2^{* * *}$ \\
\hline
\end{tabular}

Fonte: Microdados da PNAD (2002 e 2011), exclusive áreas rurais de AC, AM, AP, PA, RO e RR. Obs.: (1) Trabalhadores formais: funcionários públicos, trabalhadores com carteira assinada e empregadores.

${ }^{*} \mathrm{p}<0,10 ;{ }^{* *} \mathrm{p}<0,05 ;{ }^{* * *} \mathrm{p}<0,01$, com incorporação do desenho amostral da PNAD (Silva, Pessoa e Lila, 2002).

Todas as variações foram estatisticamente significativas. O percentual de trabalhadores formais cresceu quase 12 pontos percentuais (p.p.), a renda média real aumentou quase $22 \%$ e o número de horas trabalhadas caiu, ao mesmo tempo que a desigualdade da renda diminuiu de acordo com os três índices.

No que diz respeito à desigualdade, particularmente, é possível chegar a afirmações ainda mais fortes a partir das curvas de Lorenz para 2002 e 2011: como não há interseção entre elas, pode-se concluir inequivocamente que houve redução da desigualdade entre os dois anos não só para as três medidas enumeradas na Tabela 2 como para todas as outras medidas comumente utilizadas.

Em suma, desde que as PNADs passaram a ir a campo anualmente, em meados dos anos 1970, esta foi a primeira vez que se registrou um pe- 
ríodo prolongado de crescimento da renda do trabalho com queda da desigualdade.

A Tabela 3 apresenta a distribuição da população ocupada e remunerada segundo classes e a renda média de cada classe em 2002 e 2011. No primeiro caso, vemos que a distribuição pouco mudou. As modificações mais relevantes ocorreram nas classes mais pobres, com aumento relativo dos trabalhadores típicos e encolhimento dos elementares, contas próprias precários e empregados domésticos. No outro extremo da pirâmide social, o acesso à (grande) propriedade praticamente não variou e, analogamente, houve pouca mudança no que diz respeito às classes assalariadas mais abastadas. No geral, o índice de dissimilaridade entre as duas distribuições é pequeno: pouco mais de $7 \%$ dos casos teriam que ser reclassificados para que ambas fossem idênticas.

Quanto à renda média de cada classe, percebem-se mudanças mais expressivas. Enquanto as classes com maiores rendas em 2002 tiveram

Tabela 3

Distribuição e Renda Média das Classes da População Ocupada Remunerada (Brasil, 2002 e 2011)

\begin{tabular}{|c|c|c|c|c|c|c|}
\hline \multirow[t]{2}{*}{ Classe } & \multicolumn{2}{|c|}{ Distribuição (\%) } & \multirow{2}{*}{$\begin{array}{c}\text { Variação } \\
\text { (p.p.) }\end{array}$} & \multicolumn{2}{|c|}{ Renda (R\$ 2013) } & \multirow{2}{*}{$\begin{array}{l}\text { Varia- } \\
\text { ção (\%) }\end{array}$} \\
\hline & 2002 & 2011 & & 2002 & 2011 & \\
\hline Capitalistas e fazendeiros & 0,6 & 0,5 & $-0,1^{*}$ & 7.884 & 9.764 & $23,9 * *$ \\
\hline Pequenos empregadores & 3,9 & 2,9 & $-1,0^{* * *}$ & 3.122 & 3.360 & $7,6^{* *}$ \\
\hline Contas próprias não agrícolas & 7,4 & 7,1 & $-0,4^{* * *}$ & 1.448 & 1.723 & $19,0^{* * *}$ \\
\hline Contas próprias agrícolas & 5,9 & 4,5 & $-1,5^{* * *}$ & 523 & 749 & $43,1^{* * *}$ \\
\hline Especialistas autônomos & 1,1 & 1,2 & $0,2^{* *}$ & 4.712 & 4.557 & $-3,3$ \\
\hline Gerentes & 2,8 & 2,5 & $-0,2^{* * *}$ & 3.453 & 3.730 & $8,0^{* *}$ \\
\hline Empregados especialistas & 3,6 & 5,0 & $1,4^{* * *}$ & 3.697 & 3.859 & $4,4^{*}$ \\
\hline Empregados qualificados & 7,2 & 7,6 & $0,4^{* * *}$ & 1.547 & 1.725 & $11,4^{* * *}$ \\
\hline Supervisores & 1,8 & 1,3 & $-0,5^{* * *}$ & 1.627 & 2.279 & $40,0^{* * *}$ \\
\hline Trabalhadores típicos & 34,4 & 39,9 & $5,5^{* * *}$ & 887 & 1.107 & $24,8^{* * *}$ \\
\hline Trabalhadores elementares & 12,0 & 10,2 & $-1,8^{* * *}$ & 460 & 697 & $51,7^{* * *}$ \\
\hline Contas próprias precários & 10,9 & 9,6 & $-1,3^{* * *}$ & 644 & 937 & $45,5^{* * *}$ \\
\hline Empregados domésticos & 8,4 & 7,7 & $-0,6^{* * *}$ & 400 & 564 & $40,8^{* * *}$ \\
\hline Total & 100,0 & 100,0 & - & 1.193 & 1.454 & $21,9 * * *$ \\
\hline
\end{tabular}

Fonte: Microdados da PNAD (2002 e 2011), exclusive áreas rurais de AC, AM, AP, PA, RO e RR. Obs.: ${ }^{*} \mathrm{p}<0,10,{ }^{* *} \mathrm{p}<0,05,{ }^{* * *} \mathrm{p}<0,01$, com incorporação do desenho amostral da PNAD (Silva, Pessoa e Lila, 2002). 
flutuações menores e até não significativas - o que implica que as variações que ocorreram em alguns casos podem ser apenas um artefato estatístico - todas as classes com as piores remunerações obtiveram ganhos expressivos e estatisticamente significativos. Ou seja, enquanto a estrutura de classes pouco mudou, os salários relativos - isto é, as razões entre os rendimentos médios do trabalho de cada classe - mudaram muito mais, diminuindo a desigualdade entre classes.

Por que esse fenômeno ocorreu? Embora haja certamente diversos fatores em jogo, as mudanças no perfil educacional da força de trabalho desempenham um papel central. A distribuição por níveis educacionais e a renda média de cada nível são apresentados na Tabela 4. Ao contrário da estrutura de classes, a composição educacional dos trabalhadores ativos mudou muito: todos os níveis inferiores perderam participação relativa e todos os mais altos ampliaram-se. Todas as variações foram estatisticamente significativas, evidenciando o crescente nível educacional das gerações mais jovens. As mudanças foram particularmente intensas nos dois extremos da distribuição: o percentual de indivíduos sem escolaridade ou apenas com o elementar incompleto caiu mais de 7 p.p., enquanto o de pessoas com ensino superior com-

Tabela 4

Distribuição e Renda Média por Grupos Educacionais da População Ocupada

Remunerada

(Brasil, 2002 e 2011)

\begin{tabular}{|c|c|c|c|c|c|c|}
\hline \multirow[t]{2}{*}{ Nível educacional } & \multicolumn{2}{|c|}{ Distribuição (\%) } & \multirow{2}{*}{$\begin{array}{c}\text { Variação } \\
\text { (p.p.) }\end{array}$} & \multicolumn{2}{|c|}{ Renda (R\$ 2013) } & \multirow{2}{*}{$\begin{array}{c}\text { Variação } \\
(\%)\end{array}$} \\
\hline & 2002 & 2011 & & 2002 & 2011 & \\
\hline Ensino Superior completo & 8,4 & 13,0 & $4,6^{* * *}$ & 4.079 & 3.712 & $-9,0^{* * *}$ \\
\hline Ensino Superior incompleto & 5,2 & 7,0 & $1,9 * * *$ & 1.896 & 1.685 & $-11,1^{* * *}$ \\
\hline Ensino Médio completo & 19,8 & 28,7 & $8,9^{* * *}$ & 1.349 & 1.334 & $-1,1$ \\
\hline Ensino Médio incompleto & 8,5 & 9,1 & $0,6^{* * *}$ & 821 & 935 & $13,8^{* * *}$ \\
\hline Fundamental completo & 8,1 & 8,9 & $0,8^{* * *}$ & 1.045 & 1.145 & $9,6^{* * *}$ \\
\hline Fundamental incompleto & 16,2 & 11,5 & $-4,7^{* * *}$ & 722 & 895 & $24,0^{* * *}$ \\
\hline Elementar completo & 11,7 & 7,0 & $-4,7^{* * *}$ & 828 & 991 & $19,6^{* * *}$ \\
\hline Elementar incompleto & 15,5 & 11,2 & $-4,3^{* * *}$ & 579 & 787 & $35,9^{* * *}$ \\
\hline Sem escolaridade & 6,7 & 3,6 & $-3,1^{* * *}$ & 430 & 657 & $53,0^{* * *}$ \\
\hline Total & 100,0 & 100,0 & - & 1.193 & 1.454 & $21,9^{* * *}$ \\
\hline
\end{tabular}

Fonte: Microdados da PNAD (2002 e 2011), exclusive áreas rurais de AC, AM, AP, PA, RO e RR. Obs.: ${ }^{*} \mathrm{p}<0,10,{ }^{* *} \mathrm{p}<0,05,{ }^{* * *} \mathrm{p}<0,01$, com incorporação do desenho amostral da PNAD (Silva, Pessoa e Lila, 2002). 
pleto ou não cresceu 6,5 p.p. Com isso, pela primeira vez este último grupo se tornou mais numeroso do que o primeiro. Enquanto o índice de dissimilaridade da distribuição das 13 classes foi de 7,4\%, o da distribuição de apenas nove grupos educacionais atingiu 16,8\%.

Ao mesmo tempo, as mudanças nas remunerações médias também foram expressivas. Por um lado, a mesma tendência observada anteriormente se confirma: o crescimento da renda foi maior entre os menos escolarizados. Por outro, a renda média dos indivíduos com maior escolaridade teve uma queda estatística e substantivamente significativa, ao contrário do observado anteriormente para as classes mais privilegiadas: enquanto o rendimento médio de um trabalho com nível superior completo caiu 9\% - em função sobretudo do aumento deste grupo ao longo do tempo -, os rendimentos das classes com maior remuneração apresentaram variações em geral positivas. A razão entre a renda média de um trabalhador com ensino superior completo e outro sem escolaridade caiu $41 \%$, de 9,5 para 5,6.

Em conjunto, as Tabelas 3 e 4 mostram mudanças muito mais rápidas na distribuição da educação do que na estrutura de classes. Como argumentamos, desde sempre a educação tem sido apontada como um dos grandes determinantes da desigualdade brasileira e o próprio conceito de "qualificação" constitui um dos ativos mais relevantes na tipologia de classes utilizada. Governos quase nunca conseguem mudar deliberadamente a estrutura de classes de um país no curto prazo. A educação talvez seja o ativo produtivo mais sensível a políticas públicas, mas os resultados disso podem reverberar nas relações de classe como um todo, como se vê nas Tabelas 3 e 4 , acarretando mudanças importantes na estruturação da desigualdade.

\section{DECOMPOSIÇÕES DA DESIGUALDADE MEDIDA PELO GE(0)}

A Tabela 5 traz os valores absolutos e relativos dos componentes "entre grupos" das decomposições estáticas do GE(0) para as duas variáveis de interesse - classe e educação - e para a combinação delas em 2002 e 2011. Como discutimos anteriormente, os valores indicam quanto as diferenças entre rendas médias contribuem para a desigualdade total.

Os resultados mostram que a tipologia de classes explica um percentual maior da desigualdade do que os grupamentos educacionais tanto em 2002 quanto em 2011. Neste último ano, a desigualdade de rendimentos médios entre classes respondiam por cerca de $40 \%$ da desigual- 
dade total, contra pouco mais de $30 \%$ para a desigualdade entre níveis educacionais. Como o GE(0) pode ser interpretado contrafactualmente, esses números implicam que a desigualdade de renda do trabalho cairia $40 \%$ caso as diferenças médias entre classes fossem eliminadas e $30 \%$ caso as diferenças de remuneração entre níveis educacionais desaparecessem.

Entre 2002 e 2011, a queda nas desigualdades "entre grupos" para classe e educação foi mais rápida do que a queda na desigualdade total, de modo que o peso relativo desses componentes diminuiu 4 p.p. nos dois casos. Em termos absolutos, a queda do componente "entre grupos" para educação foi um pouco mais forte do que para classes; em termos relativos, a magnitude foi muito parecida, embora o poder explicativo da educação fosse menor inicialmente.

Como classe e educação estão correlacionadas e como as mudanças educacionais foram mais intensas do que na estrutura de classes, isso sugere a possibilidade de que apenas o componente educacional tenha de fato mudado, isto é, que toda a queda observada no componente entre classes decorra apenas de mudanças educacionais, como exploraremos mais adiante.

\section{Tabela 5}

Decomposições Estáticas do Índice GE(0) da Desigualdade por Subgrupos Populacionais: Classes, Educação e Classes+Educação

(Brasil, 2002 e 2011)

\begin{tabular}{c|c|c|c}
\hline & $\mathbf{2 0 0 2}$ & $\mathbf{2 0 1 1}$ & Variação \\
\hline Classes & & & \\
Absoluto & 0,251 & 0,177 & $-29,5^{* * *}$ \\
$\begin{array}{c}\text { Relativo } \\
\text { Educação }\end{array}$ & $44,2 \%$ & $40,2 \%$ & $-4,0$ p.p.*** \\
Absoluto & & & \\
Relativo & 0,196 & 0,134 & $-31,5^{* * *}$ \\
Classes + educação & $34,5 \%$ & $30,5 \%$ & $-4,0$ p.p. ${ }^{* * *}$ \\
Absoluto & & & $-29,2^{* * *}$ \\
Relativo & 0,289 & 0,204 & $-4,4$ p.p.p.** \\
\hline
\end{tabular}

Fonte: Microdados da PNAD (2002 e 2011), exclusive áreas rurais de AC, AM, AP, PA, RO e RR. Obs.: ${ }^{*} \mathrm{p}<0,10,{ }^{* *} \mathrm{p}<0,05,{ }^{* * *} \mathrm{p}<0,01$, com incorporação do desenho amostral da PNAD (Silva, Pessoa e Lila, 2002). 
A combinação de classe e educação também apresentou quedas absolutas e relativas. No entanto, vale observar que as variáveis educacionais acrescentam pouca informação às de classes, mas as de classes acrescentam muita informação às educacionais. Comparando a última decomposição com as duas anteriores, vê-se que a contribuição marginal da adição da educação à decomposição por classes é muito pequena, de pouco mais de 6 p.p. da desigualdade total. Já a contribuição marginal da adição das classes à decomposição por educação é muito maior, chegando a mais da metade do percentual explicado pela educação sozinha (em 2002, 50,9\%-34,5\% = 16,4\%; em 2011, 46,4\%-30,5\% = $16,0 \%)$.

Até aqui, a análise baseou-se em decomposições estáticas, isto é, nos fatores estruturantes da desigualdade em pontos específicos do tempo. Podemos também decompor a mudança entre os dois anos para avaliar a importância de cada fator, o que convencionalmente se chama de decomposição dinâmica da desigualdade.

A Tabela 6 mostra as contribuições relativas obtidas das decomposições dinâmicas do GE(0) para as variáveis de estrutura de classes, de grupos educacionais e a combinação entre ambas. Nos três casos, o efeito renda é o mais importante: o principal componente da queda da desigualdade é a redução das diferenças nos rendimentos relativos entre os grupos, confirmando o que foi visto nas Tabelas 3 e 4 . Na decomposição por classes, por exemplo, o maior percentual de crescimento da renda média do trabalho para as classes mais pobres respondeu por $52,1 \%$ da queda do GE(0), enquanto a queda da desigualdade dentro das classes somou $34,4 \%$ e as mudanças no tamanho relativo das classes ficaram com apenas $13,4 \%$.

\section{Tabela 6}

Contribuições Relativas para a Queda da Desigualdade: Decomposições Dinâmicas do Índice GE(0) por Classes, Educação e Classes+Educação (\%)

(Brasil, 2002-2011)

\begin{tabular}{l|c|c|c|c}
\hline & Efeito Puro & Efeito Alocação & Efeito Renda & $\begin{array}{c}\text { Variação do } \\
\text { Theil-L }\end{array}$ \\
\hline Classes & 34,4 & 13,4 & 52,1 & 100,0 \\
Educação & 52,6 & $-14,5$ & 61,8 & 100,0 \\
Classes + educação & 28,1 & 10,3 & 61,6 & 100,0 \\
\hline
\end{tabular}

Fonte: Microdados da PNAD (2002 e 2011), exclusive áreas rurais de AC, AM, AP, PA, RO e RR. 
O efeito "puro", ligado às desigualdades internas aos grupos, foi mais forte no caso da educação. Na prática, isso significa que tanto os grupos educacionais quanto as classes tornaram-se internamente menos desiguais, isto é, mais homogêneas, mas o ritmo foi maior no caso da educação. Contudo, como vimos na Tabela 5, as classes utilizadas já eram e permaneceram sendo mais homogêneas do que os grupos educacionais. Assim, não se deve exagerar a importância deste resultado.

Finalmente, cabe notar que, nas três decomposições, o efeito alocação é relativamente pequeno, chegando a ser negativo no caso da educação, isto é, contribuindo para um aumento da desigualdade nesta decomposição. Em suma, a mudança no tamanho relativo dos grupos educacionais entre os dois anos contribuiu para um aumento da desigualdade. Mais uma vez, não se deve dar importância desmesurada a este resultado, que era em certa medida previsível, uma vez que o efeito alocação capta apenas mudanças ceteris paribus na composição da população e houve grande aumento nos níveis educacionais com rendimentos mais elevados.

Como separar as mudanças educacionais das mudanças na estrutura de classes? Em outras palavras, como isolar a educação enquanto ativo produtivo estruturante da desigualdade no mercado de trabalho e testar se, além dessas mudanças, houve modificações correspondentes nos demais ativos que delimitam as relações de classe?

As decomposições univariadas do GE(0) ofereceram, afinal, uma primeira indicação: a variável "classes" capta mais dimensões do que a puramente educacional e, por isso, explica mais a desigualdade total; além disso, a contribuição mais forte para a queda da desigualdade vem de mudanças nos salários relativos por níveis educacionais, mais do que por classes. Isso sugere que as mudanças educacionais alteraram a relação entre educação e classe e, portanto, é possível que os demais ativos destacados pela estrutura de classes - a estrutura burocrática de organização do emprego e o acesso à propriedade - tenham na realidade permanecido mais ou menos constantes.

Na próxima seção, estendemos a análise para decomposições baseadas em modelos multivariados que nos permitem "isolar" os efeitos de cada variável em cada ano e entre os anos. Ao contrário das decomposições acima, nessas decomposições via regressão é possível investigar a influência de determinadas variáveis sobre a desigualdade mantendo constantes uma série de controles, isto é, outros fatores que também 
possivelmente afetam a distribuição de renda, mas que não são centrais para este trabalho.

\section{DECOMPOSIÇÕES VIA REGRESSÃO DA DESIGUALDADE NO MERCADO DE TRABALHO}

As decomposições multivariadas com base em regressões foram aplicadas para 2002 e 2011 em três modelos distintos, sempre com o mesmo conjunto de variáveis de controle: dummy para brancos ou amarelos; quatro dummies para as cinco macrorregiões; dummy para áreas urbanas; duas dummies para as três áreas censitárias da PNAD (municípios autorrepresentativos e não autorrepresentativos, assumindo regiões metropolitanas como categoria de referência); experiência (em anos) e experiência ao quadrado; tempo no emprego atual (em anos) e tempo no emprego atual ao quadrado; e o logaritmo das horas trabalhadas por semana. Além dessas, incluíram-se, no primeiro modelo, 12 dummies para as 13 classes discriminadas acima; no segundo, oito dummies para os nove grupos educacionais; no terceiro e último, foram incluídas tanto as dummies para educação quanto para classe. Em todas as regressões, a variável dependente foi o logaritmo natural da renda do trabalho principal em reais de 2013. Modelos alternativos foram testados, tanto no que diz respeito à variável dependente quanto às independentes, sem que os resultados tenham sido substantivamente alterados.

As decomposições estáticas com os pesos relativos das variáveis para a desigualdade em 2002 e 2011 estão na Tabela 7. As variáveis de classes mais uma vez aparecem com maior poder explicativo do que a educação, ou seja, representam maior percentual da desigualdade total do que as variáveis educacionais. Isso ocorre tanto quando comparamos os dois modelos em separado como quando olhamos para o modelo com os dois conjuntos de variáveis. Mais ainda, a queda no percentual da desigualdade explicado pela educação foi maior do que no das classes. Nos modelos em separado, o percentual da desigualdade correspondente às variáveis de classes recuou 1,8 p.p., de $28,7 \%$ para $26,9 \%$, enquanto o dos grupos educacionais caiu 4,4 p.p., de $25,9 \%$ para $21,5 \%$. Logo, mesmo partindo de percentuais mais baixos, a queda foi maior para as variáveis educacionais.

No modelo com os dois conjuntos de variáveis, o contraste é ainda mais nítido: o peso relativo das variáveis de classe fica basicamente 
constante, enquanto o da educação recua mais de 3 p.p. Se em 2002 as classes respondiam por 2,6 p.p. da desigualdade a mais do que a educação, em 2011 já eram quase 6 p.p. Os números se tornam ainda mais chamativos quando lembramos que se trata de estimativas conservadoras, uma vez que artificialmente removem todo o efeito educacional da estrutura de classes, cuja construção almeja levar precisamente o efeito "qualificação" em conta.

Tabela 7

Decomposições Estáticas da Desigualdade Via Regressão (\%)

(Brasil, 2002 e 2011)

\begin{tabular}{c|c|c|c}
\hline & $\mathbf{2 0 0 2}$ & $\mathbf{2 0 1 1}$ & Variação (p.p.) \\
\hline Classes & & & $-1,8$ \\
Classes & 28,7 & 26,9 & $-5,1$ \\
Controles $^{*}$ & 27,4 & 22,3 & 6,9 \\
Resíduo & 44,0 & 50,8 & - \\
Total & 100,0 & 100,0 & \\
Educação & & & $-4,4$ \\
Educação & 25,9 & 21,5 & $-4,5$ \\
Controles* & 27,9 & 23,4 & 8,9 \\
Resíduo & 46,2 & 55,0 & - \\
Total & 100,0 & 100,0 & 0,0 \\
Classes + educação & & & $-3,3$ \\
Classes & 19,2 & 19,3 & $-4,3$ \\
Educação & 16,6 & 13,3 & 7,6 \\
Controles* & 24,7 & 20,4 & - \\
Resíduo & 39,4 & 47,0 & $\mathbf{1 0 0 , 0}$ \\
\hline Total
\end{tabular}

Fonte: Microdados da PNAD (2002 e 2011), exclusive áreas rurais de AC, AM, AP, PA, RO e RR. Obs.: * Controles: dummies para brancos ou amarelos; macrorregiões; áreas urbanas; áreas censitárias; e logaritmos naturais das horas trabalhadas por semana, do total de anos trabalhados e do total de anos no trabalho atual.

Vale observar que esses números não significam que a desigualdade entre classes líquida da influência da educação tenha permanecido estática no período: como vimos, as contribuições absolutas das variáveis dependem dos índices específicos utilizados para medir a desigualdade. No caso do GE(0) e também de outros índices, a magnitude da queda da desigualdade total foi tão grande que é possível dizer que as contribuições absolutas de todas as variáveis diminuíram no perío- 
do. De fato, o que ocorreu é que a contribuição das variáveis de classes caiu em termos absolutos em ritmo semelhante à da desigualdade total, e, assim, seu peso relativo pouco mudou. Enquanto isso, o ritmo da queda da desigualdade entre níveis educacionais foi fulminante mais intenso do que a já significativa queda da desigualdade de renda do trabalho como um todo -, fazendo com que a desigualdade entre eles caísse bastante em termos relativos.

Se considerarmos que no último modelo a inclusão das variáveis de classe e educação permite isolar razoavelmente - ainda que artificialmente, como dissemos - os efeitos das mudanças educacionais dos demais ativos estruturantes das relações de classe e da demanda por emprego, parece lícito então interpretar os resultados como sinais de que: a) embora tenha havido progresso generalizado em termos absolutos, as mudanças educacionais foram mais rápidas do que nas demais dimensões da estrutura de classes; e b) apesar de haver espaço para o prosseguimento do processo no curto prazo, é provável que no médio e no longo prazo essa contribuição das mudanças educacionais atinja seus limites e a queda da desigualdade desacelere bastante, pois as demais dimensões da estrutura de classes e da demanda por emprego passariam a constituir gargalos para sua continuidade.

Finalmente, a Tabela 8 exibe a decomposição dinâmica da desigualdade entre 2002 e 2011 para o GE(0), seguindo o modelo de Fields. Em consonância com as evidências anteriores, as variáveis educacionais são o principal elemento por trás da queda da desigualdade. No modelo completo, a educação explica $28 \%$ da queda do GE(0), e as classes, menos de $20 \%$, reiterando a diferença de ritmo nas mudanças educa-

Tabela 8

Aplicação ao Índice GE(0) das Decomposições Dinâmicas da Desigualdade Via Regressão (\%) (Brasil, 2002 e 2011)

\begin{tabular}{l|c|c|c}
\hline & 1. Classes & 2. Educação & 3. Classes + educação \\
\hline Classes & 34,8 & - & 19,1 \\
Educação & - & 41,0 & 28,0 \\
Controles* $^{*}$ & 44,9 & 43,3 & 39,5 \\
Resíduo & 20,3 & 15,6 & 13,3 \\
\hline Total & $\mathbf{1 0 0 , 0}$ & $\mathbf{1 0 0 , 0}$ & $\mathbf{1 0 0 , 0}$ \\
\hline
\end{tabular}

Fonte: Microdados da PNAD (2002 e 2011), exclusive áreas rurais de AC, AM, AP, PA, RO e RR. Obs.: * Controles: dummies para brancos ou amarelos; macrorregiões; áreas urbanas; áreas censitárias; e logaritmos naturais das horas trabalhadas por semana, do total de anos trabalhados e do total de anos no trabalho atual. 
cionais vis-à-vis as demais dimensões captadas pela tipologia de classes. Embora não seja o foco da nossa análise, chama a atenção também o papel dos controles, que englobam sobretudo variáveis relacionadas à segmentação do mercado de trabalho - por gênero, por região, por área de moradia e afins -, o que é compatível com a literatura que destaca a maior integração e menor segmentação como elemento também importante para a queda recente da desigualdade (Ulyssea, 2007).

Os resultados parecem corroborar a interpretação formulada acima. Todas as variáveis contribuíram para a queda da desigualdade, o que aponta para a robustez e generalidade do processo. No entanto, a diminuição das diferenças entre níveis educacionais parece ter sido o grande motor do fenômeno, ao passo que as demais dimensões estruturantes da desigualdade brasileira caminharam de forma muito mais lenta. Ao menos no período em questão, a melhoria do perfil educacional da força de trabalho em um contexto de poucas mudanças estruturais na distribuição da população por classes serviu para aumentar a competição por melhores postos e comprimir os diferenciais de renda, reduzindo a desigualdade.

Do ponto de vista normativo, parece seguro afirmar que se trata de um fenômeno extremamente positivo. Afinal, os diferenciais salariais associados à desigualdade de escolaridade vêm sendo apontados há décadas como um componente estruturante da desigualdade brasileira, tanto pela literatura econômica quanto pela sociológica. $\mathrm{O}$ argumento é bem conhecido: dada a alta desigualdade educacional entre a população brasileira, haveria uma remuneração relativamente desproporcional para as credenciais de educação no mercado de trabalho, devido à escassez relativa de trabalhadores mais educados (Langoni, 1973; Ferreira, 2000; Barbosa Filho e Pessoa, 2008; Souza, Ribeiro e Carvalhaes, 2010). Nesse sentido, os avanços educacionais nas últimas duas décadas parecem ter ensejado um processo de erosão dessas "rendas" (Sorensen, 2000), aumentando a competição entre os estratos de maior escolaridade.

Dado o peso histórico da educação na desigualdade brasileira e o fato de ser uma área mais sensível a mudanças provocadas por políticas públicas do que a estrutura de classes, é compreensível que as mudanças sigam o padrão acima. No entanto, como ficou claro, em médio e longo prazos a continuidade do processo de redução das desigualdades tende a depender cada vez mais das outras dimensões aqui captadas pelas 
variáveis de classe, notadamente o acesso à propriedade e a organização burocrática do trabalho, entre outras.

\section{CONCLUSÃO}

O objetivo principal deste artigo foi analisar as mudanças ocorridas na estrutura de classes e no perfil educacional da força de trabalho brasileira na última década, de forma a esclarecer melhor o papel das classes e da educação na estruturação da desigualdade brasileira, tanto no que se refere ao nível da desigualdade quanto à sua queda no período estudado.

No que diz respeito especificamente à queda da desigualdade, uma série de explicações possíveis já foram levantadas, com destaque para aquelas influenciadas pelas características da oferta de trabalho. Nessa linha, o argumento principal ancora-se nas mudanças que aumentaram o nível de escolaridade e deixaram a força de trabalho mais homogênea em termos educacionais, o que acabou por diminuir os altos retornos para os trabalhadores mais qualificados. Sem desconsiderar esse tipo de explicação, o texto procurou investigar a questão a partir de um ponto de vista tipicamente sociológico, qual seja, o da estrutura de classes. Em particular, tratou-se de tentar entender em que medida houve mudanças em outros aspectos estruturais da desigualdade, para além da educação.

Para isso, operamos a partir da tipologia de classes de inspiração neomarxista de Santos (2005) e procuramos investigar especificamente o papel das classes e da educação. Como tal tipologia baseia-se na distribuição dos ativos produtivos e em sua liquidez em um contexto como o brasileiro, a ideia era verificar se as mudanças observadas foram provenientes apenas de avanços educacionais ou se decorreram também de mudanças relevantes na distribuição de outros ativos e na organização do trabalho.

De início, mostramos que, enquanto a distribuição da força de trabalho ocupada e remunerada por classes alterou-se pouco entre 2002 e 2011, a distribuição por níveis educacionais mudou significativamente. As decomposições univariadas estáticas do GE(0) mostraram que o percentual da desigualdade total explicado pelas classes ou grupos educacionais diminuiu no período, o que significa, nos dois casos, que a desigualdade entre grupos caiu mais rapidamente do que a desigualdade total. Ainda assim, a desigualdade entre classes continua com po- 
der explicativo maior do que a desigualdade entre grupos educacionais. No caso das decomposições dinâmicas, vimos que mudanças nas desigualdades intraclasses e intragrupos educacionais foram menos importantes do que as mudanças entre grupos para a queda da desigualdade.

Tudo isso confirma que a tipologia de classes utilizadas captura outras dimensões estruturantes da desigualdade para além da educação, mas não responde se essas dimensões também mudaram ou não. Para isso, recorremos à decomposição proposta por Fields (2003) a partir de regressões lineares. Com o auxílio de uma série de variáveis de controle, operacionalizamos um modelo apenas com variáveis para classe, outro apenas com variáveis educacionais e um terceiro com ambas.

Mais uma vez, a capacidade explicativa das variáveis de classes foi superior à das educacionais. Mais importante do que isso, no entanto, foi observar o que acontece com os pesos relativos de ambas quando controlamos pelas duas simultaneamente: enquanto o percentual da desigualdade explicado pela educação cai abruptamente, o das variáveis de classes permanece constante.

Em termos substantivos, isso aponta para duas conclusões: a) de fato o avanço educacional parece ter sido muito mais importante para a queda na desigualdade do que mudanças nas demais dimensões - acesso à propriedade, organização burocrática da demanda por trabalho, entre outros - captadas pela estrutura de classes; e b) caso esse processo continue é possível que ele atinja um limite no médio e no longo prazo, pois essas outras dimensões da estrutura de classes, que explicam parte significativa da desigualdade, tornar-se-iam entraves para seu aprofundamento.

Finalmente, as decomposições dinâmicas via regressão corroboraram tal interpretação, na medida em que, mesmo no modelo com ambas as variáveis, as mudanças educacionais contribuíram de forma muito mais relevante para a queda na desigualdade do que as mudanças na estrutura de classes. Em resumo, portanto, o aperfeiçoamento das políticas educacionais nas últimas décadas parece ter redundado de fato em um processo - necessário e positivo - de erosão das "rendas" ligadas à educação, constitutivas desde sempre da estrutura da desigualdade no Brasil. Assim, o papel da educação na estrutura de classes brasileira parece estar mudando de forma rápida. No entanto, caso o objetivo último seja o prosseguimento do processo de redução da desigual- 
dade, parece provável que outras dimensões institucionais da demanda por emprego adquiram cada vez mais importância nos próximos anos.

(Recebido para publicação em junho de 2012)

(Reapresentado em outubro de 2013)

(Aprovado para publicação em janeiro de 2014)

\section{NOTA}

1. É possível fazer decomposições multivariadas do GE(0) a partir da combinação de diversas variáveis, mas, na prática, isso é normalmente inviável pois requer amostras grandes o suficiente para impedir a existência de células vazias. 


\section{REFERÊNCIAS BIBILIOGRÁFICAS}

BARBOSA FILHO, Fernando de Holanda e PESSOA, Samuel. (2008), "Retorno da Educação no Brasil". Pesquisa e Planejamento Econômico, vol. 38, no 1, pp. 97-125.

BARROS, Ricardo Paes de, CARVALHO, Mirela, FRANCO, Samuel e MENDONÇA, Rosane. (2007), "Determinantes Imediatos da Queda da Desigualdade de Renda Brasileira", in R. P. Barros; M. N. Foguel e G. Ulyssea (orgs.), Desigualdade de Renda no Brasil: Uma Análise da Queda Recente. Brasília, Ipea, vol. 2.

BARROS, Ricardo Paes de, FRANCO, Samuel e MENDONÇA, Rosane. (2007a), "A Recente Queda na Desigualdade de Renda e o Acelerado Progresso Educacional Brasileiro da Última Década", in R. P. Barros; M. N. Foguel e G. Ulyssea (orgs.), Desigualdade de Renda no Brasil: Uma Análise da Queda Recente. Brasília, Ipea, vol. 2.

. (2007b), "Discriminação e Segmentação no Mercado de Trabalho e Desigualdade de Renda no Brasil", in R. P. Barros; M. N. Foguel e G. Ulyssea (orgs.), Desigualdade de Renda no Brasil: Uma Análise da Queda Recente. Brasília, Ipea, vol. 2.

BREEN, Richard e ROTTMAN, David. (1995), Class Stratification: A Comparative Perspective. New York, Harvester Wheatsheaf.

CORSEUIL, Carlos Henrique e FOGUEL, Miguel N. (2002), "Uma Sugestão de Deflatores para Rendas Obtidas a partir de Algumas Pesquisas Domiciliares do IBGE". Texto para Discussão no 897. Rio de Janeiro, Ipea.

ERIKSON, Robert e GOLDTHORPE, John. (1992), The Constant Flux: A Study of Class Mobility in Industrial Societies. New York, Oxford University Press.

FERREIRA, Francisco H. G. (2000), "Os Determinantes da Desigualdade de Renda no Brasil: Luta de Classes ou Heterogeneidade Educacional?", in R. Henriques (org.), Desigualdade e Pobreza no Brasil. Rio de Janeiro, Ipea.

FERREIRA, Francisco H. G., LEITE, Philippe, LITCHFIELD, Julie e ULYSSEA, Gabriel. (2006), "Ascensão e Queda da Desigualdade de Renda no Brasil". Econômica, vol. 8, no 1, pp. 147-169.

FIELDS, Gary. (2003), "Accounting for Income Inequality and Its Change: A New Method, with Application to the Distribution of Earnings in the United States". Research in Labor Economics, no 22, pp. 1-38.

FORTIN, Nicole; LEMIEUX, Thomas e FIRPO, Sérgio. (2011), "Decomposition Methods in Economics", in O. Ashenfelter e D. Card (orgs.), Handbook of Labor Economics. Amsterdam, Elsevier.

FOSTER, James e OK, Efe. (1999), "Lorenz Dominance and the Variance of Logarithms". Econometrica, vol. 67, no 4, pp. 901-907.

GOLDTHORPE, John. (2000), "Social Class and the Differentiation of Employment Contracts", in On Sociology: Numbers, Narratives, and the Integration of Research and Theory. New York, Oxford University Press.

GRUSKY, David e SORENSEN, Jesper. (1998), "Can Class Analysis Be Salvaged?" The American Journal of Sociology, vol. 103, no 5, pp. 1187-1234. 
HOFFMANN, Rodolfo. (1973), "Considerações sobre a Evolução Recente da Distribuição da Renda no Brasil". Revista de Administração de Empresas, vol. 13, no 4, pp. 7-17.

(2007), "Transferências de Renda e Redução da Desigualdade no Brasil e em Cinco Regiões, entre 1997 e 2005", in R. P. Barros; M. N. Goguel e G. Ulyssea (orgs.), Desigualdade de Renda no Brasil: Uma Análise da Queda Recente. Brasília, Ipea, vol. 2.

HOFFMANN, Rodolfo e DUARTE, João Carlos. (1972), "A Distribuição da Renda no Brasil". Revista de Administração de Empresas, vol. 12, no 2, pp. 46-66.

LANGONI, Carlos. (1973), Distribuição de Renda e Desenvolvimento Econômico do Brasil. Rio de Janeiro, Editora Expressão e Cultura.

MENEZES-FILHO, Naércio; FERNANDES, Reynaldo e PICCHETTI, Paulo. (2006), "Educação e Queda Recente da Desigualdade no Brasil", in R. P. Barros; M. N. Goguel e G. Ulyssea (orgs.), Desigualdade de Renda no Brasil: Uma Análise da Queda Recente. Brasília, Ipea, vol. 2.

MOOKHERJEE, Dilip e SHORROCKS, Anthony. (1982), "A Decomposition Analysis of the Trend in UK Income Inequality". The Economic Journal, vol. 92, no368, pp. 886-902.

PARKIN, Frank. (1979), Marxism and Class Theory: A Bourgeois Critique. New York, Columbia University Press.

SANTOS, José Alcides Figueiredo. (2005), "Uma Classificação Socioeconômica para o Brasil". Revista Brasileira de Ciências Sociais, vol. 20, no 58, pp. 27-45.

SHORROCKS, Anthony. (1980), "The Class of Additively Decomposable Inequality Measures". Econometrica, vol. 48, no 3, pp. 613-625.

. (1982), "Inequality Decomposition by Factor Components". Econometrica, vol. 50, no 1 , pp. 193-211.

SILVA, Pedro Luis do N.; PESSOA, Djalma Galvão C. e LILA, Maurício F. (2002), "Análise Estatística de Dados da PNAD: Incorporando a Estrutura do Plano Amostral". Ciência E Saúde Coletiva, vol. 7, no 4, pp. 659-670.

SOARES, Sergei. (2006), "Distribuição de Renda no Brasil de 1976 a 2004 com Ênfase no Período entre 2001 e 2004". Texto para Discussão, (1166), no 31: Rio de Janeiro, Ipea.

SORENSEN, Aage B. (1996), "The Structural Basis of Social Inequality". The American Journal of Sociology, vol. 101, no 5, pp. 1333-1365.

. (2000), "Towards a Sounder Basis for Class Analysis". The American Journal of Sociology, vol. 105, no 6, pp. 1523-1558.

SORENSEN, Aage B. e KALLEBERG, Arne. (1981), "An Outline for a Theory of the Matching of Persons to Jobs", in I. Berg (org.), Sociological Perspectives on Labor Markets. New York, The Academic Press. pp. 49-74.

SOUZA, Pedro H. G. F., RIBEIRO, Carlos Antônio C. e CARVALHAES, Flavio. (2010), "Desigualdade de Oportunidades no Brasil: Considerações sobre Classe, Educação e Raça". Revista Brasileira de Ciências Sociais, vol. 25, no 73, pp. 77-100.

ULYSSEA, Gabriel. (2007), "Segmentação no Mercado de Trabalho e Desigualdade de Rendimentos no Brasil: Uma Análise Empírica", in R. P. Barros; M. N. Goguel e G. 
Ulyssea (orgs.), Desigualdade de Renda no Brasil: Uma Análise da Queda Recente. Brasília, Ipea, vol. 2.

WEEDEN, Kim. (2002), "Why Do Some Occupations Pay More than Others? Social Closures and Earnings Inequality in the United States". The American Journal of Sociology, vol. 108, no 1, pp. 55-101.

WRIGHT, Erik O. (2000), Class Counts. Cambridge, Cambridge University Press.

(2005), "Foundations of a Neo-Marxist Class Analysis", in E. O. Wright (org.), Approaches to Class Analysis. Cambridge, Cambridge University Press.

\section{ABSTRACT \\ Class Structure, Education and the Reduction in Income Inequality (2002-2011)}

This paper aims to reconcile the role ascribed to education as a major cause of the recent reduction in inequality in Brazil with a class analysis framework. A series of univariate and multivariate inequality decompositions were carried out, including the between-group/within-group decomposition of the GE(0) index and the regression-based decomposition suggested by Fields (2003). Our results show that a) whereas the class structure composition was relatively stable between 2002 and 2011, the educational composition changed dramatically; b) inequality between classes accounts for a larger fraction of total inequality than inequality between educational groups; and c) when class and education are analyzed simultaneously, the fraction of total inequality explained by education falls abruptly, while the relative share of class inequality remains constant. Thus, educational improvement was apparently much more important for the reduction in inequality than changes in the other dimensions captured by class structure.

Key words: class structure; income inequality; educational inequality 


\section{RÉSUMÉ}

Structure des Classes Sociales, Éducation et Diminution de l'Inégalité des Revenus (2002-2011)

Dans cet article, on cherche à rapprocher l'importance attribuée à l'éducation comme cause de la récente diminution des inégalités au Brésil de l'optique de l'analyse des classes sociales. Dans ce but, on a procédé à une série de décompositions de l'inégalité des revenus du travail, y compris la décomposition du $\mathrm{GE}(0)$ et la décomposition via régression. Les résultats montrent que: a) la composition de la force de travail en termes de classes n'a guère changé entre 2002 et 2011, alors que la composition concernant l'éducation a présenté une altération significative; b) la typologie de classes utilisée explique mieux le niveau d'inégalités que celle des groupes d'éducation; c) quand on analyse simultanément classes sociales et éducation, on se rend compte que le pourcentage d'inégalités expliqué par l'éducation tombe brutalement, tandis que celui des classes sociales reste relativement constant. Bref, le progrès en éducation semble avoir été bien plus important pour la diminution des inégalités que les changements dans les autres secteurs pris en compte par la structure de classes, qui ont progressé très lentement.

Mots-clés: structure de classes; inégalités des revenus; inégalités dans l'éducation

\section{RESUMEN}

Estructura de Clases, Educación y Reducción de la Desigualdad de Ingresos (2002-2011)

El objetivo de este texto es combinar el destaque dado a la educación como causa de la reciente reducción de la desigualdad en Brasil con el punto de vista del análisis de clases. Para ello, fueron realizadas una serie de descomposiciones de la desigualdad de los ingresos laborales, incluso la descomposición del GE(0) y la descomposición vía regresión. Los resultados muestran que: a) la composición de la fuerza de trabajo en términos de clases cambió poco entre 2002 y 2011, pero la composición educativa se modificó significativamente; b) la tipología de clases empleada explica mejor el nivel de desigualdad que los grupos educativos; c) cuando se analiza simultáneamente clases y educación, se percibe que el porcentaje de desigualdad explicado según la educación se reduce abruptamente, pero el de las clases permanece relativamente constante. En definitiva, el avance educativo parece haber sido mucho más importante para la reducción de la desigualdad que los cambios en las demás dimensiones captadas por la estructura de clases, que avanzaron muy lentamente.

Palabras clave: estructura de clases; desigualdad de ingresos; desigualdad educativa 